\title{
BMJ Open Association of apolipoprotein Cs with new-onset type 2 diabetes mellitus: findings from the Chinese multi- provincial cohort study
}

\author{
Jiangtao Li, Dong Zhao (D) , Jing Liu, Miao Wang, Jiayi Sun, Jun Liu, Yan Li (D) , \\ Qiuju Deng, Yue Qi (D)
}

To cite: Li J, Zhao D, Liu J, et al. Association of apolipoprotein Cs with new-onset type 2 diabetes mellitus: findings from the Chinese multi-provincial cohort study. BMJ Open 2021;11:e052388. doi:10.1136/ bmjopen-2021-052388

- Prepublication history and additional supplemental material for this paper are available online. To view these files, please visit the journal online (http://dx.doi.org/10.1136/ bmjopen-2021-052388)

Received 14 April 2021 Accepted 25 October 2021

Check for updates

(c) Author(s) (or their employer(s)) 2021. Re-use permitted under CC BY-NC. No commercial re-use. See rights and permissions. Published by BMJ.

Department of Epidemiology, Beijing An Zhen Hospital, Capita Medical University, Beijing Institute of Heart, Lung and Blood Vessel Diseases, The Key Laboratory of RemodelingRelated Cardiovascular Diseases, Ministry of Education, Beijing Municipal Key Laboratory of Clinical Epidemiology, Beijing, China

Correspondence to

Dr Yue Qi; qiyue_bjcn@163.com

\section{ABSTRACT}

Objectives Apolipoprotein Cs (apoCs), especially apoCII and apoC-III, as the components of triglyceride-rich lipoproteins, play a key role in the pathophysiology of diabetes. However, prospective studies examining direct associations between apoCs and diabetes are not reproducible. The aim of this study was to evaluate the impact of apoCs on the risk of developing diabetes in a middle-aged population, and to explore possible mediators responsible for the relationship between apoCs and diabetes.

Design Prospective cohort study.

Setting Community-based study carried out in Beijing. Methods ApoCs were measured in 1085 participants aged $45-74$ years and free of type 2 diabetes mellitus (T2DM) at baseline from the Chinese Multi-Provincial Cohort Study-Beijing Project. Multivariate logistic regression was performed to examine the association of apoCs with a 5-year risk of new-onset T2DM. The impacts of triglycerides, insulin and high-sensitivity $C$ reactive protein (hs-CRP) on the association between apoC-III and the risk of T2DM were explored by a mediation test. Results During the 5 years of follow-up, 97 (8.9\%) participants developed T2DM. ApoC-III was significantly associated with the risk of developing T2DM after multivariable adjustment ( $\mathrm{OR}=1.40 ; 95 \% \mathrm{Cl} 1.07$ to 1.82$)$. This association was mainly mediated by triglyceride levels with a significant indirect effect (OR 1.61; $95 \% \mathrm{Cl}$ 1.19 to 2.18 ), followed by hs-CRP and insulin.

Conclusions Our findings demonstrated that higher serum apoC-III was independently associated with increased 5-year risk of new-onset T2DM in the Chinese population, and triglyceride plays a crucial role in mediating this relationship. More attention should be paid to preventive strategies of T2DM targeting apoC-III.

\section{INTRODUCTION}

Diabetes is an important cause of morbidity and mortality, leading to heavy disease and economic burdens worldwide, especially in low-income and middle-income countries. ${ }^{1}$ In China, diabetes has reached epidemic proportions, ${ }^{2}$ with $11.6 \%$ of adults suffering from diabetes, accounting for an estimated
Strengths and limitations of this study

- This is the first study that investigated the association between apolipoprotein Cs (apoCs) levels and risk of diabetes among the Chinese population from a large community-based cohort study.

- The mediation analysis was conducted to explore the possible mediators responsible for the relation between apoC-III and diabetes.

- Further intervention studies are warranted to confirm the causal relationship between apoC-III and diabetes.

- The impact of apoC-III on diabetes may be underestimated due to partial differences between participants who were eligible for the final analyses and those who were unavailable for the re-examination.

103 million adults, and about $24 \%$ of the global number of patients with diabetes, ${ }^{3}$ and China is among the countries with the highest diabetes prevalence in Asia and has the largest burden of diabetes worldwide. ${ }^{4}$ However, current preventive strategies primarily targeting glucose homoeostasis have failed to mitigate the growing burden. Consequently, identifying novel risk factors related to diabetes is urgently needed to further the understanding of the aetiology and prevention of the disease.

More recent studies have found that lipid-related pathways are involved in regulating glucose homoeostasis, suggesting the possibility of targeting lipid metabolism to prevent diabetes. Previous experimental studies suggested that apolipoprotein Cs (apoCs), especially apoC-II and apoC-III, as the components of triglyceride-rich lipoproteins, have a great influence on the lipolysis of triglycerides, ${ }^{5}$ as well as the ability to regulate insulin signalling, pancreatic beta-cell apoptosis and inflammation. ${ }^{6-8}$ These studies provided pathophysiological evidence for the 
critical role of apoCs in the development of diabetes. To date, five prospective cohort studies ${ }^{9-13}$ have reported on the association of apoC-II and apoC-III levels with diabetes risk, whereas the results were not often reproducible after controlling for triglycerides, leaving open the question of whether the apoCs are indeed associated with diabetes risk, and whether this association is dependent on triglycerides, as well as insulin and inflammation. Furthermore, most of the studies on apoCs and diabetes have been conducted in European or American populations, while the evidence from Asian populations is scarce.

Whether the levels of apoCs are indeed related to diabetes risk is critically important and relevant to diabetes prevention strategies. Therefore, the aim of this study was to evaluate the impact of serum apoCs levels on the risk of developing type 2 diabetes mellitus (T2DM) in a middle-aged community-based cohort of Chinese participants. In addition, this study was also aimed to investigate the mediation effects of triglyceride, insulin, and highsensitivity $\mathrm{C}$ reactive protein (hs-CRP) on the association between apoCs and T2DM in light of the underlying physiological functions of apoCs involved in the development of diabetes.

\section{METHODS}

\section{Study design and population}

Study participants were recruited from the Chinese MultiProvincial Cohort Study-Beijing Project, a populationbased prospective cohort study. The details of study design and initial exclusion criteria have been described elsewhere. ${ }^{14}$ Briefly, 1392 non-diabetic participants aged 45-74 years completed questionnaires on demographic characteristics and measurements of traditional risk factors in 2002. All participants were followed up to identify any occurrence of cardiovascular diseases every 1-2 years from baseline and invited to a repeated examination to identify risk factors in 2007. After excluding participants for the following reasons: unavailable blood samples $(n=134)$, death of causes other than T2DM $(n=33)$, and loss to follow-ups $(n=140)$, totally 1085 participants were enrolled for this study (online supplemental figure 1). Written informed consents were obtained from all participants.

\section{Risk factor surveys}

Demographic and clinical characteristics were collected via standardised questionnaires in all the surveys, including demographic characteristics, personal and family medical history, medical therapy, smoking status, and alcohol consumption.

Anthropometric measurements, including height and weight, and blood pressure, were obtained by trained physicians during physical examination. Body mass index (BMI) was calculated as weight in kilograms divided by height in metres squared. Blood pressure was calculated by averaging two consecutive recordings, measured at the right-side brachial artery with the participants in a sitting position using a mercury sphygmomanometer after resting for at least $5 \mathrm{~min}$. Current smoking was defined as smoking one or more cigarettes per day for more than 3 months. Current drinking was defined as drinking at least once a month for more than 6 months. Overweight was defined as having a BMI of $25 \mathrm{~kg} / \mathrm{m}^{2}$ or higher. ${ }^{15}$ Hypertension was defined as systolic blood pressure $\geq 140 \mathrm{~mm}$ $\mathrm{Hg}$, diastolic blood pressure $\geq 90 \mathrm{~mm} \mathrm{Hg}$ and/or current antihypertensive treatment. Physical activity was defined as at least 150 min of moderate-intensity physical activity or 75 min of high-intensity physical activity (or equivalent combination of moderate-intensity and high-intensity physical activity) every week.

\section{Laboratory measurements}

Venous blood samples were collected for laboratory measurements after fasting for at least 8 hours. Total cholesterol, low-density lipoprotein cholesterol, highdensity lipoprotein cholesterol, fasting blood glucose $(\mathrm{FBG})$, and triglyceride were measured using fresh samples on the day of collection using the same method in all the surveys. Total cholesterol, FBG and triglyceride levels were tested by enzymatic methods (Human Diagnostics, Wiesbaden, Germany). Low-density lipoprotein cholesterol and high-density lipoprotein cholesterol levels were measured using a homogeneous method (Daiichi, Tokyo, Japan). The remaining samples were stored at $-80^{\circ} \mathrm{C}$ without repeated freeze-thaw cycles to minimise the degradation. Fasting insulin levels were determined by chemiluminescent immunoassay in 2008. Hs-CRP levels were determined using a particle-enhanced immunoturbidimetric method (DiaSys, Holzheim, Germany) in 2008. Baseline ApoCs levels were tested by immunoturbidimetric method (Sekisui Medical, Tokyo, Japan) in 2015. The coefficient of variation (CV) of apoC-II was $1.29 \%$ for low-range controls and $2.68 \%$ for high-range controls. The CV of apoC-III was $1.67 \%$ for low-range controls and $2.30 \%$ for high-range controls. Insulin resistance was estimated by homoeostasis model assessment of insulin resistance (HOMA-IR), which was calculated as the formulae: HOMA-IR=fasting insulin $(\mu \mathrm{IU} / \mathrm{mL}) \mathrm{FBG}$ $(\mathrm{mmol} / \mathrm{L}) / 22.5 .^{16}$

\section{Case ascertainment}

New-onset T2DM was determined if any of the following criteria were met: (1) previously diagnosed by a physician; (2) use of insulin or glucose-lowering medications during the past 2 weeks; (3) FBG $\geq 126 \mathrm{mg} / \mathrm{dL}$; (4) death of T2DM during the follow-up visits that was established by the staff from collaborating centres and by regular searching of the death registration database of Beijing. ${ }^{17}$

\section{Sample size estimation}

The risk estimates of apoC-III for T2DM reported in previous studies were 1.42-3.43. In our study, the rate of new-onset T2DM during follow-up was $8.94 \%$. The SD of apoC-III was 4.23 . $\mathrm{R}^{2}$ value for apoC-III with other T2DM risk factors was 0.15 . The estimated maximum sample 
size was 74-922, assuming an alpha (probability of type I error) of 0.05 and a delta (admissible error) of 0.20 . The actual sample size of 1085 enabled sufficient statistical power.

\section{Statistical analyses}

Continuous variables were expressed as means $( \pm \mathrm{SD})$ in the case of normal distributions or as medians (IQR), and compared using unpaired Student's t-test or MannWhitney U test, as appropriate. Categorical variables were expressed as numbers (percentages) and compared using the $\chi^{2}$ test. Correlations between the levels of apoCs and other parameters were estimated using Spearman's rank method after adjusting age and sex.

OR for new-onset T2DM associated with apoCs were calculated using the logistic regression model after adjustment for traditional metabolic risk factors and variables different between participants with and without new-onset T2DM, including age, sex, parental history for diabetes, overweight, physical activity, hypertension, current smoking, current drinking, lipid-lowering treatment, lowdensity lipoprotein cholesterol, high-density lipoprotein cholesterol, hs-CRP and FBG. Risk estimates were calculated for $1 \mathrm{SD}$ increases in naturally log-transformed apoCs. Because participants did not undergo the oral glucose tolerance test at baseline, some participants with T2DM that was only identified by the oral glucose tolerance test were included, which was particularly the case among participants with impaired fasting glucose (IFG) . Therefore, a sensitivity analysis was performed among participants without IFG (FBG $>6.1 \mathrm{mmol} / \mathrm{L})$ at baseline. Subgroup analyses were performed using baseline characteristics, including sex (male or female), age $(<60$ or $\geq 60$ years), parental history of diabetes (no or yes), overweight (no or yes), current smoking (no or yes), current drinking (no or yes), physical activity (no or yes), hypertension (no or yes), triglyceride ( $<150$ or $\geq 150 \mathrm{mg} / \mathrm{dL}$ ),

Table 1 Baseline characteristics of study participants

\begin{tabular}{|c|c|c|c|}
\hline & $\begin{array}{l}\text { Non-T2DM } \\
(\mathrm{n}=988)\end{array}$ & $\begin{array}{l}5 \text {-year new-onset T2DM } \\
(\mathrm{n}=97)\end{array}$ & P value $^{*}$ \\
\hline Age (years) & $59 \pm 8$ & $60 \pm 8$ & 0.234 \\
\hline Female, n (\%) & $552(55.9)$ & $45(46.4)$ & 0.073 \\
\hline Parental history of diabetes, $\mathrm{n}(\%)$ & $103(10.4)$ & $13(13.4)$ & 0.365 \\
\hline Body mass index, $\left(\mathrm{kg} / \mathrm{m}^{2}\right)$ & $24.69 \pm 3.11$ & $26.92 \pm 3.30$ & $<0.001$ \\
\hline Overweight, n (\%) & $439(44.4)$ & $68(70.1)$ & $<0.001$ \\
\hline Systolic blood pressure $(\mathrm{mm} \mathrm{Hg})$ & $128 \pm 18$ & $138 \pm 17$ & $<0.001$ \\
\hline Diastolic blood pressure $(\mathrm{mm} \mathrm{Hg})$ & $80 \pm 10$ & $85 \pm 10$ & $<0.001$ \\
\hline Hypertension, n (\%) & $475(48.1)$ & $67(69.1)$ & $<0.001$ \\
\hline Total cholesterol (mg/dL) & $213.00(189.00-237.00)$ & $215.00(193.50-249.50)$ & 0.305 \\
\hline HDL cholesterol (mg/dL) & $53.00(45.00-62.00)$ & $48.00(41.50-53.50)$ & $<0.001$ \\
\hline LDL cholesterol (mg/dL) & $128.00(108.25-148.00)$ & $127.00(100.50-152.50)$ & 0.867 \\
\hline Triglycerides (mg/dL) & $114.00(82.00-164.00)$ & $158.00(106.50-232.50)$ & $<0.001$ \\
\hline Fasting blood glucose (mg/dL) & $83.00(79.00-89.00)$ & $95.00(88.00-104.00)$ & $<0.001$ \\
\hline Fasting insulin (ulU/mL) & $6.1(4.5-8.3)$ & $8.2(5.9-10.6)$ & $<0.001$ \\
\hline HOMA-IR & $1.27(0.91-1.76)$ & $1.96(1.30-2.58)$ & $<0.001$ \\
\hline $\mathrm{Hs}-\mathrm{CRP}$ (mg/L) & $0.77(0.36-1.67)$ & $1.58(0.74-3.73)$ & $<0.001$ \\
\hline Current smoking, $\mathrm{n}(\%)$ & $110(11.1)$ & $13(13.4)$ & 0.501 \\
\hline Current drinking, $\mathrm{n}(\%)$ & $291(29.5)$ & $26(26.8)$ & 0.584 \\
\hline Physical activity, n (\%) & $263(26.6)$ & $33(34.0)$ & 0.122 \\
\hline Prevalent cardiovascular disease, $\mathrm{n}(\%)$ & $43(4.4)$ & $3(3.1)$ & 0.557 \\
\hline Lipid-lowering treatment, n (\%) & $102(10.3)$ & $18(18.6)$ & 0.014 \\
\hline Apolipoprotein C-II (mg/dL) & $3.92(2.69-5.43)$ & $4.56(3.06-6.72)$ & 0.001 \\
\hline Apolipoprotein C-III (mg/dL) & $9.80(7.53-12.55)$ & $11.74(8.60-15.63)$ & $<0.001$ \\
\hline
\end{tabular}

Data are expressed as number (per cent) for categorical variables and as mean (SD) for continuous variables in case of normal distributions and median (IQR) otherwise.

*Unpaired Student's t-test (when satisfying a normal distribution) or Mann-Whitney U test (when not satisfying a normal distribution) for quantitative variables and $\chi^{2}$ test for qualitative variables.

HDL, high-density lipoprotein; HOMA-IR, homoeostasis model assessment of insulin resistance; hs-CRP, high-sensitivity $\mathrm{C}$ reactive protein; LDL, low-density lipoprotein; T2DM, type 2 diabetes mellitus; VLDL, very-low-density 3 lipoprotein. 
Table 2 ORs of new-onset T2DM associated with the levels of apolipoprotein Cs

\begin{tabular}{|c|c|c|c|c|}
\hline & \multicolumn{2}{|l|}{$\begin{array}{l}\text { All participants } \\
(n=1085)\end{array}$} & \multicolumn{2}{|c|}{$\begin{array}{l}\text { Participants without IFG at baseline } \\
(n=1069)\end{array}$} \\
\hline & OR (95\% Cl) & $P$ value & OR (95\% Cl) & $P$ value \\
\hline \multicolumn{5}{|c|}{ Apolipoprotein C-II } \\
\hline Unadjusted & 1.54 (1.22 to 1.95$)$ & $<0.001$ & 1.54 (1.20 to 1.98$)$ & $<0.001$ \\
\hline Model 1 & 1.54 (1.22 to 1.95$)$ & $<0.001$ & 1.56 (1.21 to 2.00$)$ & $<0.001$ \\
\hline Model 2 & 1.34 (1.04 to 1.73$)$ & 0.023 & 1.30 (1.00 to 1.69$)$ & 0.054 \\
\hline Model 3 & $1.40(1.08$ to 1.81$)$ & 0.011 & 1.36 (1.04 to 1.79$)$ & 0.026 \\
\hline Model 4 & 1.25 (0.95 to 1.66$)$ & 0.117 & 1.21 (0.90 to 1.62$)$ & 0.203 \\
\hline \multicolumn{5}{|c|}{ Apolipoprotein C-III } \\
\hline Unadjusted & 1.66 (1.33 to 2.08$)$ & $<0.001$ & 1.65 (1.30 to 2.08 ) & $<0.001$ \\
\hline Model 1 & $1.71(1.36$ to 2.14$)$ & $<0.001$ & 1.70 (1.34 to 2.16$)$ & $<0.001$ \\
\hline Model 2 & 1.54 (1.21 to 1.96$)$ & $<0.001$ & 1.48 (1.15 to 1.91$)$ & 0.002 \\
\hline Model 3 & 1.59 (1.25 to 2.03$)$ & $<0.001$ & 1.54 (1.19 to 1.99$)$ & 0.001 \\
\hline Model 4 & 1.40 (1.07 to 1.82$)$ & 0.013 & 1.40 (1.06 to 1.84$)$ & 0.019 \\
\hline
\end{tabular}

Model 1: adjusted for age (per 1 year), sex and parental history for diabetes.

Model 2: model 1+ overweight, hypertension, current smoking and current drinking, physical activity, lipid-lowering treatment, low-density lipoprotein cholesterol levels (per $1 \mathrm{mg} / \mathrm{dL}$ ) and, high-density lipoprotein cholesterol (per $1 \mathrm{mg} / \mathrm{dL}$ ).

Model 3: model 2+ naturally log-transformed high-sensitivity $\mathrm{C}$ reactive protein.

Model 4: model 3+ fasting blood glucose levels (per $1 \mathrm{mg} / \mathrm{dL}$ ).

The ORs and corresponding Cls for 1 SD increases in naturally log-transformed apolipoprotein C-II or apolipoprotein C-III were calculated using the logistic regression model.

IFG, impaired fasting glucose; T2DM, type 2 diabetes mellitus.

high-density lipoprotein cholesterol $(<40$ (males) $/ 50$ (females) or $\geq 40$ (males) $/ 50$ (females) $\mathrm{mg} / \mathrm{dL}$ ), lipidlowering treatment (no or yes), hs-CRP $(<3$ or $\geq 3 \mathrm{mg} / \mathrm{L}$ ), FBG $(<100.8$ or $\geq 100.8 \mathrm{mg} / \mathrm{dL})$, HOMA-IR $(<1.3$ or $\geq 1.3)$ and apoC-II $(<3.95$ or $\geq 3.95 \mathrm{mg} / \mathrm{dL})$ in a multivariable regression model. Besides the grouping factors, other factors were used as confounding variables. ORs between subgroups were compared using a $Z$ statistic. ${ }^{18}$

The impacts of triglycerides, insulin and hs-CRP on the association between apoC-III and the risk of T2DM were explored by a mediation analysis using PROCESS in SPSS. ${ }^{19}{ }^{20}$ In mediation models, baseline apoC-III levels were the independent variable $(\mathrm{X})$; new-onset T2DM was the dependent variable $(\mathrm{Y})$; and triglycerides, insulin and hs-CRP were the mediator variables (M). The following three statistical equations illustrate the associations between these variables:

1. $\mathrm{Y}=c \mathrm{X}+\mathrm{e}_{1}+$ confounders.

2. $\mathrm{M}=a \mathrm{X}+\mathrm{e}_{2}+$ confounders.

3. $\mathrm{Y}=c \mathrm{X}+b \mathrm{M}+\mathrm{e}_{3}+$ confounders.

The coefficient $c$ of equation 1 is the total effect of $\mathrm{X}$ on $\mathrm{Y}$; the coefficient $a$ of equation 2 is the effect of $\mathrm{X}$ on $\mathrm{M}$; the coefficient $b$ of equation 3 is the effect of $\mathrm{M}$ on $\mathrm{Y}$ after controlling for $\mathrm{X}$; the coefficient $c$ ' of equation 3 is the effect of $\mathrm{X}$ on Y after controlling for $\mathrm{M}$. The indirect effect, indicating the effect of $\mathrm{X}$ on $\mathrm{Y}$ through $\mathrm{M}$, was calculated as the product of coefficients $a b$ and was estimated from 5000 bootstrap samples. The bootstrap method was used to test the significance of the indirect effect. ${ }^{19}$ The $10 \%$ change-in-estimate methods were used to select the main confounding factors, including age, sex and parental history for diabetes, which may be related to apoC-III level, T2DM and mediator variables, but are unlikely to be intermediate variables in the causal pathway of these relationships. Additional variables including overweight, hypertension, current smoking, current drinking, physical activity, lipid-lowering treatment, low-density lipoprotein cholesterol and high-density lipoprotein cholesterol are potential intermediates between apoC-III and newonset T2DM, which could dilute the relationship if erroneously adjusted for. Before conducting the mediation analysis, these variables were compared between participants with and without new-onset T2DM. Sensitivity analyses were conducted to further adjust for variables, which were found to be significantly different between participants with and without new-onset T2DM.

Furthermore, to test whether missing data would introduce potential bias, comparisons were performed between participants who were eligible for the final analyses and those who were unavailable for the re-examination (online supplemental table 1), revealing no significant differences with respect to lipid and glucose-related biomarkers.

Statistical analyses were performed using the R software (V.3.6.2, R Foundation for Statistical Computing) and IBM SPSS Statistics for Windows software, V.23.0. A $\mathrm{p}<0.05$ on the two-sided test was considered statistically significant. Sample size estimation was calculated using the PASS software (V.11.0, NCSS). 


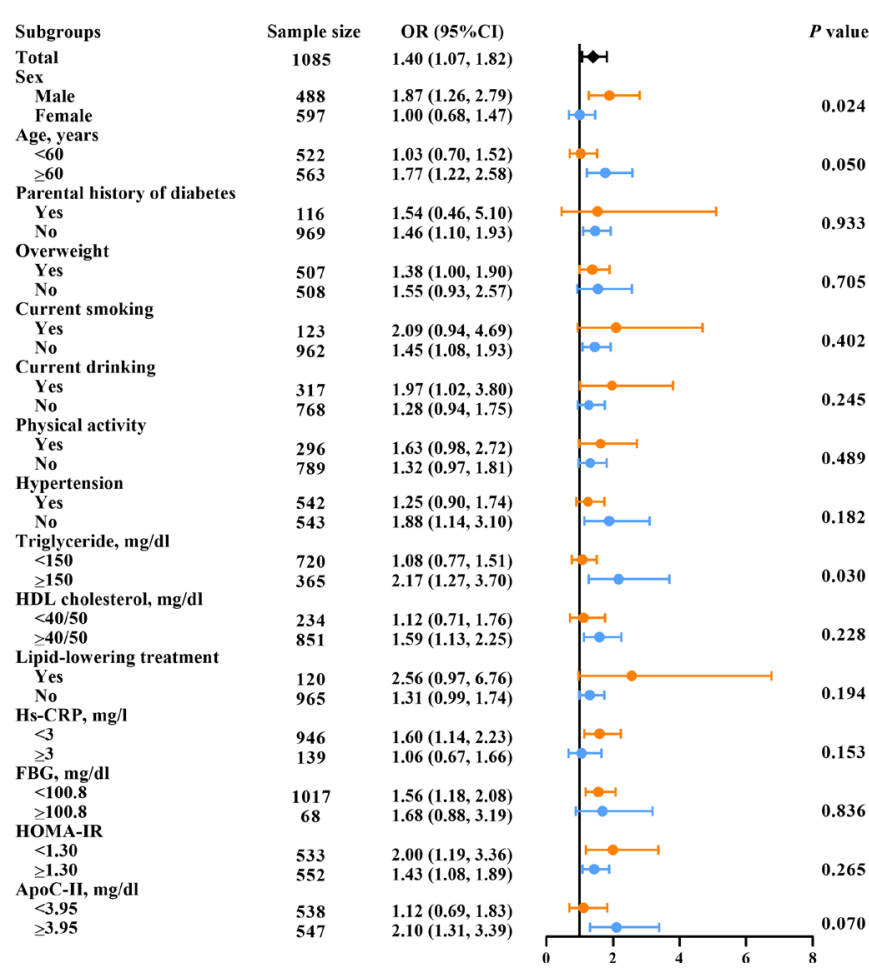

Figure 1 Subgroup analyses for the association between apolipoprotein C-III levels and new-onset T2DM. ORs are per $1 \mathrm{SD}$ naturally log-transformed, after adjusting for baseline age (per 1 year), sex, overweight, hypertension, parental history for diabetes, current smoking, current drinking, physical activity, lipid-lowering treatment, lowdensity lipoprotein cholesterol levels (per $1 \mathrm{mg} / \mathrm{dL}$ ), highdensity lipoprotein cholesterol (per $1 \mathrm{mg} / \mathrm{dL}$ ), naturally logtransformed hs-CRP and fasting blood glucose levels (per 1 $\mathrm{mg} / \mathrm{dL}$ ), except where an adjusting variable was itself tested. ${ }^{*} \mathrm{P}$ value is from the test for the difference between the two ORs derived from subgroup analysis using $Z$ statistic. ApoCII, apolipoprotein C-II; FBG, fasting blood glucose; HDL, high-density lipoprotein; HOMA-IR, homoeostasis model assessment of insulin resistance; hs-CRP, high-sensitivity $C$ reactive protein; T2DM, type 2 diabetes mellitus.

\section{Patient and public involvement}

Patients or the public were not involved in this study.

\section{RESULTS}

\section{Baseline characteristics}

The 5-year rate of new-onset T2DM was $8.9 \%$ among the participants with a mean $( \pm \mathrm{SD})$ age of $59( \pm 8)$ years old. The baseline characteristics of the study participants are shown in table 1 . Those who developed new-onset T2DM during follow-up were more likely to have higher apoCII, apoC-III, BMI, blood pressure, triglycerides, FBG, fasting insulin, HOMA-IR, and hs-CRP levels, as well as a higher proportion of participants using lipid-lowering treatment than those free of T2DM (table 1). Serum apoCs levels were correlated with lipids-related, glucoserelated and inflammation-related parameters, especially with triglycerides (partial-r: 0.760 for apoC-II; 0.810 for apoC-III) (online supplemental table 2). These relations remained significant after excluding participants with lipid-lowering treatment.

\section{Association of serum apoCs levels with the risk of new-onset T2DM}

Table 2 displays the ORs and 95\% CIs for the association between levels of serum apoCs and the risk of new-onset T2DM. After adjusting for age, sex, and parental history for diabetes, baseline apoC-II (OR 1.54; 95\% CI 1.22 to 1.95 ) and apoC-III (OR 1.71; 95\% CI 1.36 to 2.14) were both significantly and positively associated with the risk of new-onset T2DM. The OR values almost remained unchanged after adjustment for other traditional risk factors. However, only apoC-III was still significantly associated with the risk of developing T2DM after further adjustment for FBG. The OR and 95\% CI for new-onset T2DM per one SD naturally log-transformed apoC-III was 1.40 (95\% CI 1.07 to 1.82). After further excluding participants with IFG, the association between levels of serum apoCs and the risk of new-onset T2DM was similar (table 2). The relationship between apoC-III and T2DM risk was investigated among various subgroups stratified by cardiovascular risk factors. There were significant interactions between apoC-III and sex, and between apoC-III and triglycerides on new-onset T2DM (figure 1).

\section{Mediation analysis for the impact of ApoC-III on the risk of new-onset T2DM}

In mediation analysis, the relationship of apoC-III to diabetes was shown to be mediated by triglyceride, insulin and hs-CRP (table 3). After adjusting for age, sex and parental history for diabetes, the total effect of apoC-III on the incidence of diabetes was 1.71 (95\% CI 1.36 to 2.14). The indirect effect through triglyceride was 1.61 (95\% CI 1.19 to 2.18), followed by hs-CRP and insulin, which had the indirect effect OR of 1.05 (95\% CI 1.01 to $1.10)$ and 1.04 (95\% CI 1.02 to 1.09 ), respectively. The results were similar for models before and after adjusting for additional variables significantly related to new-onset T2DM separately, including hypertension, overweight, lipid-lowering treatment and low levels of high-density lipoprotein cholesterol.

\section{DISCUSSION}

In this population-based cohort study, we carefully investigated the association between serum apoCs and the risk of developing diabetes. Our results showed that serum apoC-III was independently and positively associated with a 5-year risk of new-onset diabetes. Furthermore, the relationship between apoC-III and new-onset diabetes was mediated mainly by triglycerides.

The available literature on the association between apoC-III and T2DM risk ${ }^{9-13}$ is inconsistent, which may be due to differences in study populations, measurement of lipids, and multivariable-adjusted models. First, the mean age of participants in the Rotterdam Study was $73( \pm 7.5)$ years, and $51.4 \%$ of patients with coronary heart disease 
Table 3 Mediation analyses of the relationship between apoC-III and new-onset T2DM through triglycerides, insulin and hsCRP

\begin{tabular}{|c|c|c|c|c|c|c|}
\hline \multirow[b]{2}{*}{ Mediators } & \multirow{2}{*}{$\begin{array}{l}\text { Effect of apoC-III } \\
\text { on diabetes* }\end{array}$} & \multicolumn{5}{|l|}{ OR $(95 \% \mathrm{Cl})$} \\
\hline & & Model 1 & Model 2 & Model 3 & Model 4 & Model 5 \\
\hline Triglyceride & Direct effect & 1.00 (0.67 to 1.48$)$ & 1.01 (0.68 to 1.51$)$ & $1.07(0.72$ to 1.61$)$ & 0.99 (0.67 to 1.47$)$ & 1.04 (0.69 to 1.57$)$ \\
\hline \multirow[t]{2}{*}{ Insulin } & Direct effect & 1.64 (1.31 to 2.06$)$ & 1.62 (1.28 to 2.03$)$ & 1.60 (1.27 to 2.00$)$ & 1.60 (1.27 to 2.03$)$ & 1.59 (1.27 to 1.99$)$ \\
\hline & Indirect effect & 1.04 (1.02 to 1.09$)$ & $1.03(1.01$ to 1.08$)$ & $1.02(1.00$ to 1.05$)$ & 1.04 (1.01 to 1.08$)$ & $1.03(1.01$ to 1.07$)$ \\
\hline
\end{tabular}

Model 1: adjusted for age (per 1 year), sex, and parental history for diabetes.

Model 2: model 1+ hypertension.

Model 3: model 1+ overweight.

Model 4: model 1+ lipid-lowering treatment.

Model 5: model 1+ low levels of high-density lipoprotein cholesterol ( $<40 \mathrm{mg} / \mathrm{dL}$ for males, $<50 \mathrm{mg} / \mathrm{dL}$ for females).

The ORs and corresponding Cls for 1 SD increases in naturally log-transformed apoC-II or apoC-III were calculated using the logistic regression model.

*The indirect effect indicated the effect of apoC-III on new-onset diabetes through mediators, and the direct effect referred to the effect of apoC-III on new-onset diabetes after excluding the indirect effect.

apoC-III, apolipoprotein C-III; hs-CRP, high-sensitivity C reactive protein; T2DM, type 2 diabetes mellitus.

were included in the Danish Diet, Cancer and Health (DCH) study, which may bring about the higher risk of developing T2DM and strong relationship between apoC-III levels and new-onset T2DM. Second, the lipid and lipoproteins in the DCH study were measured in a non-fasting state and were subjected to more variability than fasting lipids. Finally, the relation between apoC-III and T2DM risk was different before and after adjusting for triglycerides in previous studies. ${ }^{9-12}$ Considering these issues, we investigated the association between apoC-III in the fasting state and new-onset T2DM with a generally healthy population with a mean age of 59 (range from 45 to 74) years and found that high apoC-III levels were associated with increased risk of new-onset T2DM by raising triglyceride levels, which is in line with previous studies that reported on the modified effect of triglycerides on the association between apoC-III and T2DM risk. Those studies reported that apoC-III was strongly correlated with triglycerides. The eliminated risk estimates of apoC-III with new-onset T2DM after adjustment for triglycerides reported in previous prospective studies, ${ }^{9-12}$ suggested that triglycerides might modulate the relationship between apoC-III and T2DM. Using mediation analysis, we found a significant indirect effect on the relation between apoC-III and new-onset T2DM by triglycerides. Even after additional adjustment for baseline hs-CRP, blood glucose and other risk factors, the results were unchangeable. The mediation of triglycerides may be explained by the ability of apoC-III to increase triglyceride levels by delaying and preventing the clearance of triglyceride-rich lipoproteins ${ }^{7}$ and promoting very-low-density lipoprotein assembly and secretion, ${ }^{21}$ which is the critical role of apoC-III in modulating glucose metabolism. Genetic studies of APOC3 lossof-function variants, showing the association with lower levels of triglycerides and a reduced risk of cardiovascular disease, ${ }^{22}{ }_{23}$ supported our findings. Furthermore, the
EPIC-Norfolk Study reported that the robust association of elevated apoC-III levels and coronary artery disease risk could be likely explained by apoC-III's association with triglyceride elevation, ${ }^{24}$ which indicated the proatherogenic effect of apoC-III might be mainly attributed to triglycerides. Although we showed strong correlations between apoC-III levels, T2DM risk and triglycerides, it should be stressed that this does not imply causality, and reported findings should be further confirmed by intervention studies. Currently, apoC-III targeted therapies are in the development stage, which the antisense oligonucleotide volanesorsen ${ }^{25}$ and AKCEA-APOCIII- $\mathrm{L}_{\mathrm{RX}}{ }^{26}$ showing the most promise to date. This antisense oligonucleotide resulted in apoC-III reductions up to $93.1 \%$, which translated to equally lowering of triglyceride (up to $-77.8 \%$ ). The strong impact of triglyceride on the association between apoC-III and T2DM we observed in the current study holds promise for the potential of these therapies to lower T2DM risk, which needs to be further tested by future trials.

Furthermore, apoC-III may also have an important role in carbohydrate homoeostasis through other possible mechanisms beyond its involvement in triglyceride metabolism, such as proinflammatory effect and impairment insulin signalling. ${ }^{68}$ In line with these findings, our results demonstrated that high levels of apoC-III may also increase the risk of developing diabetes through insulin and hs-CRP. However, the indirect effects of insulin and inflammation are much lower than that of triglycerides, indicating the diabetogenic effect of apoC-III mainly via triglycerides. In addition, a significant interaction between sex and apoC-III on the developing diabetes was found, and apoC-III resulted as a better indicator of diabetes in males than in females. Consistent with our findings, genetic evidence suggested that $A P O C 3$ genetic variants were associated with disturbed glucose 
homoeostasis and an unfavourable lipid profile only in non-diabetic males ${ }^{27-29}$ whereas no significant associations were observed in females. Nevertheless, the exact mechanisms in females are not fully understood, and need to be addressed by further studies.

This study has several possible limitations that should be pointed out. First, information on some potential confounding factors, such as 2-hour oral glucose tolerance or haemoglobin A1c, were unavailable. However, after excluding participants with IFG, the association between apoCs and T2DM did not change. Second, although reasonably large, the sample size prevented a more detailed classification of participants and identification of possible mediators in subgroup analyses. Third, we did not measure apoC-III levels in specific lipoprotein subclasses and specifically in high-density lipoprotein; thus, we could not investigate the association between T2DM risk and apoC-III defined high-density lipoprotein particle. Finally, some significant differences were found between the eligible participants and participants unavailable for re-examination in this study. Participants who were unavailable for re-examination were more likely to be older, males, have higher levels of hs-CRP and have higher proportions of parental history of diabetes and hypertension than eligible participants. Exclusion of these individuals may lead to an underestimation of the results of this study, as a stronger relationship between apoC-III level and risk of diabetes was found among males and those age $\geq 60$ years shown in the subgroup analyses in this study.

In conclusion, our study demonstrated that apoC-III was independently associated with a 5-year risk of newonset T2DM in the Chinese population. In addition, triglyceride plays a crucial role in mediating the relationship between apoC-III and new-onset T2DM. Our finding should be further verified by other large prospective studies, and may inform the new direction and novel target for T2DM treatment and prevention.

Acknowledgements We gratefully acknowledge the contribution of all the
investigators from participating centres in the CMCS study for data collection.

Contributors $Y Q$, JinL and DZ contributed to initial concepts and study design. JiaL and $Y Q$ analyzed data and drafted the manuscript. All authors contributed to the acquisition or interpretation of data. MW, YL and JuL contributed to data acquisition. $\mathrm{QD}$ and JS contributed to laboratory measurements. YQ, JinL and DZ contributed to manuscript revision and supervision. All authors approved the final version of the manuscript.

Funding This study was supported by the Beijing Natural Science Foundation (No. 7212006); the National Natural Science Foundation of China (grant numbers 82073635, 81570409, 81170266, and 81070226); Beijing Nova Programme Interdisciplinary Cooperation Project (grant number Z191100001119017); the National Key Research and Development Programme of China (grant number 2016YFC0900902); and the National Science and Technology Pillar Program (grant numbers 2011BAI09B01, 2011BAl11B03, 2006BAl01A01 and 2006BAI01A02).

Disclaimer The sponsors had no role in the design or conduct of the study.

Competing interests None declared.

Patient consent for publication Not applicable.

Ethics approval The study was approved by the Ethics Committee of Beijing An Zhen Hospital, Capital Medical University (the ethical approval number: ks2019029).
Provenance and peer review Not commissioned; externally peer reviewed.

Data availability statement № data are available.

Supplemental material This content has been supplied by the author(s). It has not been vetted by BMJ Publishing Group Limited (BMJ) and may not have been peer-reviewed. Any opinions or recommendations discussed are solely those of the author(s) and are not endorsed by BMJ. BMJ disclaims all liability and responsibility arising from any reliance placed on the content. Where the content includes any translated material, BMJ does not warrant the accuracy and reliability of the translations (including but not limited to local regulations, clinical guidelines, terminology, drug names and drug dosages), and is not responsible for any error and/or omissions arising from translation and adaptation or otherwise.

Open access This is an open access article distributed in accordance with the Creative Commons Attribution Non Commercial (CC BY-NC 4.0) license, which permits others to distribute, remix, adapt, build upon this work non-commercially, and license their derivative works on different terms, provided the original work is properly cited, appropriate credit is given, any changes made indicated, and the use is non-commercial. See: http://creativecommons.org/licenses/by-nc/4.0/.

\section{ORCID iDs}

Dong Zhao http://orcid.org/0000-0002-3239-3449

Yan Li http://orcid.org/0000-0003-1032-2292

Yue Qi http://orcid.org/0000-0002-6709-7041

\section{REFERENCES}

1 Seuring T, Archangelidi O, Suhrcke M. The economic costs of type 2 diabetes: a global systematic review. Pharmacoeconomics 2015;33:811-31.

$2 \mathrm{Xu} \mathrm{Y}$, Wang L, He J, et al. Prevalence and control of diabetes in Chinese adults. JAMA 2013;310:948-59.

3 NCD Risk Factor Collaboration (NCD-RisC). Worldwide trends in diabetes since 1980: a pooled analysis of 751 population-based studies with 4.4 million participants. Lancet 2016;387:1513-30.

4 International Diabetes Federation. IDF diabetes atlas. 9th edn, 2019. https://www.diabetesatlas.org/en/>

5 Jong MC, Hofker MH, Havekes LM. Role of ApoCs in lipoprotein metabolism: functional differences between APOC1, APOC2, and APOC3. Arterioscler Thromb Vasc Biol 1999;19:472-84.

6 Lee H-Y, Birkenfeld AL, Jornayvaz FR, et al. Apolipoprotein CIII overexpressing mice are predisposed to diet-induced hepatic steatosis and hepatic insulin resistance. Hepatology 2011;54:1650-60.

7 Christopoulou E, Tsimihodimos V, Filippatos T, et al. Apolipoprotein CIII and diabetes. is there a link? Diabetes Metab Res Rev 2019;35:e3118.

8 Zewinger S, Reiser J, Jankowski V, et al. Apolipoprotein C3 induces inflammation and organ damage by alternative inflammasome activation. Nat Immunol 2020;21:30-41.

9 Huth C, von Toerne C, Schederecker F, et al. Protein markers and risk of type 2 diabetes and prediabetes: a targeted proteomics approach in the KorA F4/FF4 study. Eur J Epidemiol 2019;34:409-22.

10 Brahimaj A, Ligthart S, Ikram MA, et al. Serum levels of apolipoproteins and incident type 2 diabetes: a prospective cohort study. Diabetes Care 2017;40:346-51.

11 Aroner SA, Furtado JD, Sacks FM, et al. Apolipoprotein C-III and its defined lipoprotein subspecies in relation to incident diabetes: the multi-ethnic study of atherosclerosis. Diabetologia 2019;62:981-92.

12 Aroner SA, Yang M, Li J, et al. Apolipoprotein C-III and high-density lipoprotein subspecies defined by apolipoprotein $\mathrm{C}$-III in relation to diabetes risk. Am J Epidemiol 2017;186:736-44.

13 Onat A, Hergenç G, Ayhan E, et al. Serum apolipoprotein C-III in high-density lipoprotein: a key diabetogenic risk factor in Turks. Diabet Med 2009;26:981-8.

14 Qi Y, Fan J, Liu J, et al. Cholesterol-overloaded HDL particles are independently associated with progression of carotid atherosclerosis in a cardiovascular disease-free population: a community-based cohort study. J Am Coll Cardiol 2015;65:355-63.

15 WHO Expert Consultation. Appropriate body-mass index for Asian populations and its implications for policy and intervention strategies. Lancet 2004;363:157-63.

16 Matthews DR, Hosker JP, Rudenski AS, et al. Homeostasis model assessment: insulin resistance and beta-cell function from fasting plasma glucose and insulin concentrations in man. Diabetologia 1985;28:412-9.

17 Gao S, Zhao D, Qi Y, et al. The association between serum ferritin levels and the risk of new-onset type 2 diabetes mellitus: a 10-year 
follow-up of the chinese multi-provincial cohort study. Diabetes Res Clin Pract 2017;130:154-62.

18 Altman DG, Bland JM. Interaction revisited: the difference between two estimates. BMJ 2003;326:219.

19 Preacher KJ, Hayes AF. SPSS and SAS procedures for estimating indirect effects in simple mediation models. Behav Res Methods Instrum Comput 2004;36:717-31.

20 Korsiak J, Tranmer J, Day A, et al. Sleep duration as a mediator between an alternating day and night shift work schedule and metabolic syndrome among female hospital employees. Occup Environ Med 2018;75:132-8.

21 Sundaram M, Zhong S, Bou Khalil M, et al. Expression of apolipoprotein C-III in McA-RH7777 cells enhances VLDL assembly and secretion under lipid-rich conditions. J Lipid Res 2010:51:150-61.

22 , Crosby J, Peloso GM, et al, TG and HDL Working Group of the Exome Sequencing Project, National Heart, Lung, and Blood Institute. Loss-of-function mutations in APOC3, triglycerides, and coronary disease. N Engl J Med 2014;371:22-31.

23 Jørgensen AB, Frikke-Schmidt R, Nordestgaard BG, et al. Loss-offunction mutations in APOC3 and risk of ischemic vascular disease. N Engl J Med 2014;371:32-41.
24 van Capelleveen JC, Bernelot Moens SJ, Yang X, et al. Apolipoprotein C-III levels and incident coronary artery disease risk: the EPIC-Norfolk prospective population study. Arterioscler Thromb Vasc Biol 2017;37:1206-12.

25 Zhang J, Rocha NdeA, McCullough PA. Contribution of ApoCIII to diabetic dyslipidemia and treatment with volanesorsen. Rev Cardiovasc Med 2018;19:13-19.

26 Alexander VJ, Xia S, Hurh E, et al. N-acetyl galactosamineconjugated antisense drug to APOC3 mRNA, triglycerides and atherogenic lipoprotein levels. Eur Heart J 2019;40:2785-96.

27 Russo GT, Meigs JB, Cupples LA, et al. Association of the Sst-I polymorphism at the APOC3 gene locus with variations in lipid levels, lipoprotein subclass profiles and coronary heart disease risk: the framingham offspring study. Atherosclerosis 2001;158:173-81.

28 Pérez-Jiménez F, López-Miranda J, Gómez P, et al. The SSTI polymorphism of the apo C-III gene is associated with insulin sensitivity in young men. Diabetologia 2002;45:1196-200.

29 Waterworth DM, Talmud PJ, Luan J, et al. Variants in the APOC3 promoter insulin responsive element modulate insulin secretion and lipids in middle-aged men. Biochim Biophys Acta 2003;1637:200-6. 